\title{
Robotic Sketching: A Study on Robotic Clay 3D Printing
} of the Iberoamerican Society of Digital Graphics Medellin | Colombia

\author{
Paniz Farrokhsiar \\ Pennsylvania State University | USA | puf69@psu.edu \\ Benay Gursoy \\ Pennsylvania State University | USA | bug61@psu.edu
}

\begin{abstract}
Digital fabrication tools are typically employed to materialize a fixed design. Design limits the choice of material; Natural material behavior may consider as flaws in the fabrication. What if these tools and material behaviors being used as sketching tools to generate new design ideas? In this paper, we present a workflow in which digital fabrication tools, specifically robotic arms, are used as sketching tools. It is called robotic sketching; The goal is to sketch with effects of fabrication settings on emerging behaviors of materials in first steps of design. We exemplify this workflow with a case on robotic clay 3D printing.
\end{abstract}

Keywords: digital fabrication, sketching, additive manufacturing, 3D printing with clay, Robotic

\section{INTRODUCTION}

Architects typically use digital fabrication tools only after they finalize digital designs. Once the digital representation of design parts is complete, they translate their design ideas to machine language for fabrication. After several stages of trial and error with the material and tool's settings, eventually the material follows the digital order it has received. In this case, material is forced to obey the digitally produced form. Here, the material has no say in the design process. As Ingold (2009) mentions, in such situations, there is "no flow" in the process of making, and the object is not "alive."

What if instead of trying to have complete control over the material behavior and create a flawless object, natural behaviors of the materials become part of the design process? This would open up ways to explore forms that are so complex that they cannot be easily represented via abstract representations, such as drawings or 3D computer models?

Based on these assumptions, the questions we explore in this study are "Can making through digital fabrication be considered a form of sketching?" and, if so, "How can digital fabrication tools become sketching tools?" In this paper, we present the first steps of a case study on robotic clay 3D printing using an ABB 2400 robotic arm and an open-source paste extruder to exemplify a workflow in which these materials, fabrication tools and techniques could become platform for design ideation.

\section{BACKGROUND}

David Pye (1968) exemplifies "printed writing" and "writing with a pen" and compares these as "worksmanship of certainty" and "worksmanship of risk." In his definition, "worksmanship of certainty" corresponds to production processes that yield products with same appearance and features, as in mass production. Digital fabrication tools are typically used with similar aims to materialize designs as close as possible to their digital representations in the computer. On the other hand, "workmanship of risk" is closer to craft processes. In craft processes, there is no guarantee that two craft products will be the same. However, as Pye (1968) mentions, these uncertain features in using a pen instead of a printer makes the writing unique. In this project, we postulate that it is possible for digital fabrication processes to be "worksmanships of risk:" Mechanical paste extruder is considered a substitute for pen; clay a substitute for ink that leaves traces of different movements of designer's hand on the surface; and designer's hand is replaced by the robotic arm holding the extruder as a conceptual pen.

Digital fabrication tools, specifically robotic arms, are relatively new in the field of architecture. The focus in applying robotic systems in architecture is changing from an "engineering-oriented" approach to a "design-oriented" one. While the former approach uses these machinery to reduce human labor and optimize the cost and time of manufacturing and construction, the latter brings about more opportunities in exploring features that are hard to imagine, represent and simulate using computers alone (Bonwetsch, 2015)

Robotic arms allow more control and precision in the fabrication process and thus give the designers the opportunity to explore more design possibilities. Moreover, $3 \mathrm{D}$ printing enables the creation of more complex forms. Architects take advantage of the various potentials of these tools and techniques to prototype and make complex designs that are hard to fabricate using conventional methods. This opens up new ways for novel design explorations.

Clay, as a material, has been used extensively in architectural design throughout history. Clay's features in making are multifaceted: 1) its material features make it compatible for various forms and fabrication processes, 2) as a construction material, clay has been used both with conventional and digital fabrication methods, 3) it is possible to manipulate it before, during, and after the process of making, 4) its consistency can be adjusted by 
adding water to the mixture or letting it dry for a while to lose the water content. The relatively adjustable time that takes for clay to dry makes it an excellent material for exploring and sketching as it enables the designer to create an object and manipulate it to learn from its behavior. This is why we have chosen clay as the material in our robotic sketching in design workflow.

Design deals with the non-existing (Cannaerts, 2009). The vague and fuzzy nature of sketches enables the designers to explore the unknown during the design process. How these vague representations are interpreted are strictly personal, and this is what makes sketching a creative process. Architects and designers sketch not only to represent their ideas but also to generate new ones. As Lawson (2006) similarly points out, the ambiguous nature of sketches, and the uncertainties of the sketching process give architects opportunities to see unexpected elements in their own design process, which in return help them to generate new ideas.

In this paper, our aim is to extend the definition of design sketching to include digital fabrication processes along with more conventional modes of sketching. In a precedent study, Tokac et al. (2019) have previously defined a "material sketching" workflow using a robotic arm to cut incisions on cardboards. Along the same lines, we argue that similar to conventional modes of sketching, during digital fabrication processes, it is possible to see new shapes emerging from material behavior and tool affordances. Sometimes these emergent shapes are so complex that it is almost impossible to easily represent them in an abstract digital model. Therefore, working directly with digital fabrication tools during the design process can open up ways for new creative discoveries.

\section{LITERATURE REVIEW}

With the aim of identifying a suitable methodology for this research, we conducted an extensive literature review on projects that involve 3D printing paste-like materials. All the reviewed projects are analyzed from three main aspects: 1) objective / function defining the aim of the project, 2) method used by researchers to organize their exploration, 3) print setup / parameters that they prioritized on their exploration. Each of these aspects also become limitations for the other aspects.

\section{OBJECTIVES}

The objective can be interpreted as the aim of each research. The objective is being defined by the function of design, and it has a direct relation with the geometrical configuration of the design. Most of the reviewed projects took advantage of the $3 \mathrm{D}$ printing process to generate complex geometries. The reviewed projects could be divided into two categories of large-scale and small-scale. Large-scale projects are mostly modular structures, and each module is 3D printed separately from the others. The complexity in these projects could be seen in two layers: one appears on the overall form of the design; in other cases, it is in the details and small parts that make the overall form together. These details could be the texture of the printed components or design of the joints in between modules. Therefore, the reviewed projects can be categorized as projects where the focus is on the design of the overall geometrical configuration and projects where the focus is on the geometry of the detailed parts. The modules can be produced in one round of continuous 3D printing. From the geometric perspective the module category consists of tiles (2D or 2.5D) or blocks (3D).

"Woven Clay" project by Friedman (2014), "Clay Nonwoven" project by Rosenwasser (2017), "Robo Sense 2.0" project by Bilotti (2018), and panels designed by Emerging Objects as part of the projects "Seed Stitches" (2016) and "G-Code clay" (2016) are some examples of 2D panels attached on a structure to cover the façade of a building.

"Cool Brick" by Emerging Objects (2015), "Guiding Instabilities" (Shi, Cho, Taylor, \& Corre, 2019) and "Ceramic Components" (Anton \& Abdelmahgoub, 2018) projects are among the projects where the aim is to design self-standing and self-supporting architectural structures by $3 \mathrm{D}$ printing clay.

"Ceramic Morphologies" (Seibold, Hinz, del Castillo y López, Alonso, \& Mhatre, 2018), "Ceramic Information Pavilion" (Lange, Holohan, \& Kehne, Ceramic INformation Pavilion, 2017)), and "Ceramic Constellation" (Lange, Holohan, \& Kehne, Ceramic Constellation| Robotically Printed Brick Specials., 2018) are examples of large-scale projects using a substructure to assemble the modules produced by 3D printing to create architectural elements and spaces.

The most crucial factor here is that all these projects are either using clay 3D printing methods to get the desired complexity, which fulfills the objective of the research.

\section{METHODOLOGIES}

Among the explored methodologies of similar projects, three approaches are distinguishable: bottom-up, top-down and a mixed approaches. In the bottom-up approach, designers focus on the material behavior resulting from different fabrication setups. In this approach the focus is more on the emergent material behavior during fabrication rather than the designed artifact. Zach Cohen's "Hold-Up" project can be an example for the bottom-up exploration (Cohen, 2018), in which he took a specific approach towards extruding material using "machine delay" to create structures and textures of a wall, column and corner as simple architectural elements. Instead of extruding a continuous bead of material, Cohen developed a dripping system that he called "pointillistic time-based deposition", by embedding different kinds of delays in the fabrication process. These delays are inevitable but when used intentionally it is possible to control and regulate the results to design a fully functional architectural element.

Second approach is the top-down method. This is a more conventional approach to fabrication. It starts with a fixed digital design, and proceeds through processes of material optimization to materialize the exact digital form as the outcome. In this approach the abstract model defines the whole fabrication process and the desired goal.

Third approach defines a feedback loop between the bottom-up and top-down approaches. In this approach, process defines many transitions from abstract to material and vice versa. The outputs of each step will be inputs for the next steps. While the bottom-up material exploration enables the designer to learn from material behavior and 
make use of this knowledge while designing, the top-down design exploration defines the geometry and features of a final design. In other words, in the mixed approach the results of bottom-up exploration are implemented as design features to develop a design through a top-down process. This saves time and efforts in the process of material optimization and design. In the reviewed projects that adopt a mixed method of bottom-up and top-down, designers use a feedback loop to create printing toolpaths that are shaped through material behavior.

Designers of the "Woven Clay" (Friedman, Kim, \& Mesa, 2014) and "Clay Non-Woven" (Rosenwasser, Mantell, \& Sabin, 2017) projects have explored textiles as a reference to create thin tiles using material behavior, and different curved surfaces as a reference, to create different layers of transparency and unique tiles. A similar approach to design transparent tiling is presented in "RoboSense 2.0" project with a different approach of dealing with material complexity. Bilotti et al. (2018), in their paper "RoboSense2.0" studied two features of "looping" and "bridging" behavior of clay when extruding clay from a distance, and systematically explored how clay behaves and what are the results when variables are changed. They used these features to design tiles with specific textures and patterns that are very complex to be modeled digitally in the computer.

\section{PARAMETERS}

Setting up the $3 \mathrm{D}$ printing process is the most challenging part of all the projects, and it defines both limitations and potentials for further developments. The printing setup can be divided into physical and digital setup. The physical setup gets affected by the environment and the printer itself. Environmental parameters include two categories of environmental conditions such as temperature and humidity, and physical environment like the printing surface. The effects of environmental conditions on different clay bodies differ based on clay types and mixtures. Clay type defines the characteristic of each clay and by changing the water content or adding additives to clay it is possible to manipulate these characteristics and create different features.

Parameters related to the printer are divided into two categories: 1) digital, and 2) physical. Digital parameters are the inputs defining the toolpath of the print which can be defined as layer height, number of the target points and the shape of the geometry. Moreover, print setup is another category of digital inputs, which includes but are not limited to moving speed, extrusion speed and delays on each target point.

This categorization of parameters helped us to scope the exploration of this research and be able to design a systematic exploration of the desired workflow.

\section{METHODOLOGY}

In order to define the robotic sketching workflow, a show case of clay $3 \mathrm{D}$ printing was selected. The goal is to explore the possibilities to sketch with robotic clay $3 \mathrm{D}$ printing. For setting up this workflow we designed a set of exploration with the material and the tool.
A mixed methodology of bottom-up and top-down approaches has been adopted in this case of sketching with robotic clay 3D printer. Incorporating material behavior as part of the design process needs a constant transition between abstract and material environments. In the context of this research, abstract environment refers to the digital design or to the work done in the computer. A systematic material exploration, in which designer is able to learn from each transition and extract rules defining the effects of each transition, is realized. While the generated rules make it possible for designers to recreate each effect, focusing on the relationships between abstract representations and material representations, their similarities and differences enables a deeper understanding of the fabrication process that involves information and feedback from the tool (robotic clay extruder) and material (clay) (Figure 1).

The human designer plays an important role in this process as all the extracted rules and observations from this feedback loop are personal interpretations of the designer. This personal interpretation illustrates the sketching nature of the whole workflow. In this workflow designer starts his/her design with a simple toolpath as an input and set of parameters that they can systematically manipulate and check the difference between abstract and material models among all different physical objects created based on the same toolpath

In this process the final design of a product comes out as a combination of features from digital and physical models. While the input digital model can be simple and basic, the output physical model can emerge as a complex form in terms of changing shape, thickness and texture throughout the whole module.

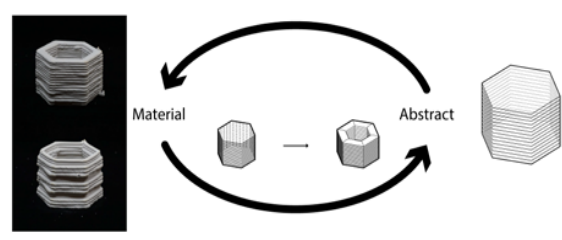

Figure 1: Constant transitions between abstract and materia environments

\section{TOOL DEVELOPMENT}

To conduct these experiments and explorations, there was a need to develop a tool that could give enough control over the process, so that it could be used as a sketching tool. The type of control needed was mostly for the real time changes in parameters such as extrusion speed rate and robot's moving speed, as designer decides what those parameters are while $3 \mathrm{D}$ printing. An open-source mechanical paste extruder (Cera, 2018) has been modified and built, along with an electronic kit that we developed to run the extruder (Figure 2). This enabled to gain control over extruder's performance and to sync it with robot's movements. 
1. Nozzle Adaptor

\section{Chamber}

3. Plunger

4. Worm-Gear Adaptor

5. Worm-Gear Reducer (Gearbox)

6. Plasric Spacer

7. Stepper Motor

8. Lead Screw Adaptor

9. Lead Screw

10. Lead Screw Guide

11. Extruder Clamp

12. Micro stepper driver

13. Mounting Board

14. Electrical Kit

15. Tool Changer

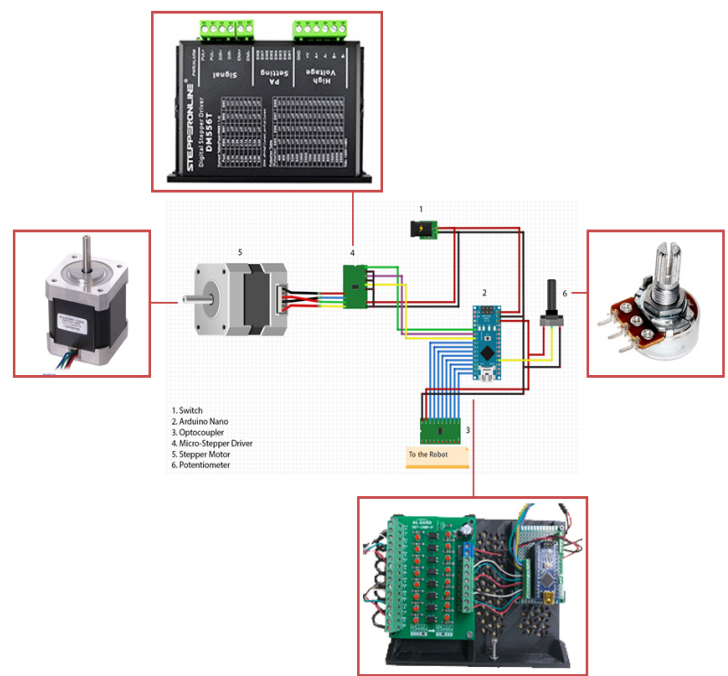

6
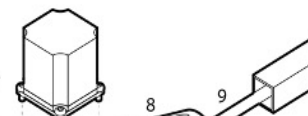

- 1 (5) 4 的

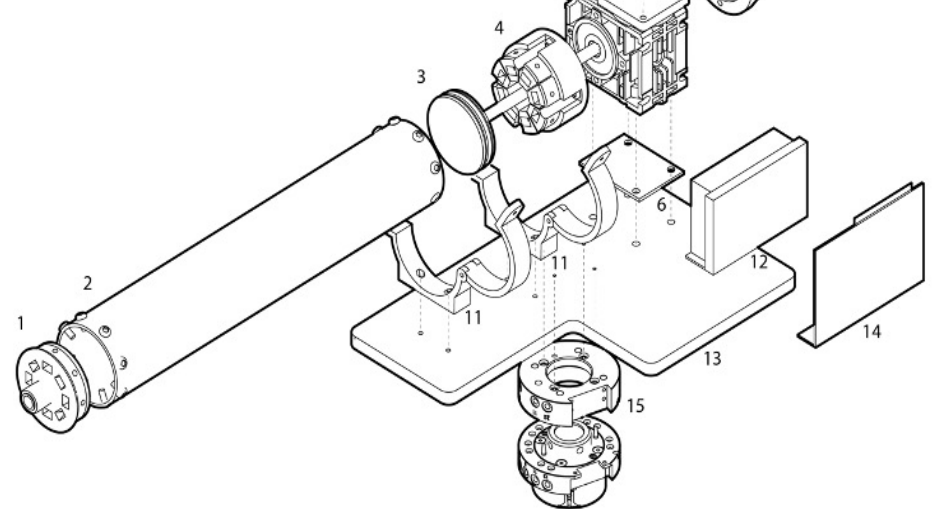

Figure 2: Exploded view of the mechanical extruder and its parts

Figure 3: Electric kit for real time control of the extruder via robot's code.

The software workflow setup includes 3D modeling in Rhinoceros 3D, generating the toolpath in Grasshopper, generating RAPID code using Robots plugin for Grasshopper, and debugging, simulating, and uploading the RAPID code to the robot using ABB Robot Studio. Additionally, Arduino Nano in the electronic kit made it possible to control the extruder's performance through the
RAPID code and in real-time using the robot's teaching pendant.

\section{BOTTOM-UP MATERIAL AND TOOL EXPLORATION}

In this research, bottom-up exploration is defined as a series of systematic explorations by changing specific parameters to observe their effects on the outcome. As a 
result of the literature review on similar projects, certain key parameters are identified and categorized based on the material and tool's features.

To begin with, three types of clay were tested in this research to explore the effects of different clay types and clay consistency on the 3D printing process and the printed products. Aneto 3D (with porcelain) and PRAI 3D (White Stoneware Clay with Impalpable Grog) are the 3D printing compatible clays. PRGM (Toasted Brown Sculpture/Raku Clay with Coarse Grog) is the third clay selected for this experiment.

The next set of parameters is defined based on 3D printing settings and setups. Printer related parameters can be categorized as digital and physical parameters. Among the digital parameters the ones selected are the Layer Height, Toolpath, Print Angle, Extrusion Speed, Moving Speed and Delays. In addition to these, Nozzle Diameter is a parameter that we altered during production. These parameters can also be categorized according to their respective effect in each step from preparation to production as shown in Figure 4.
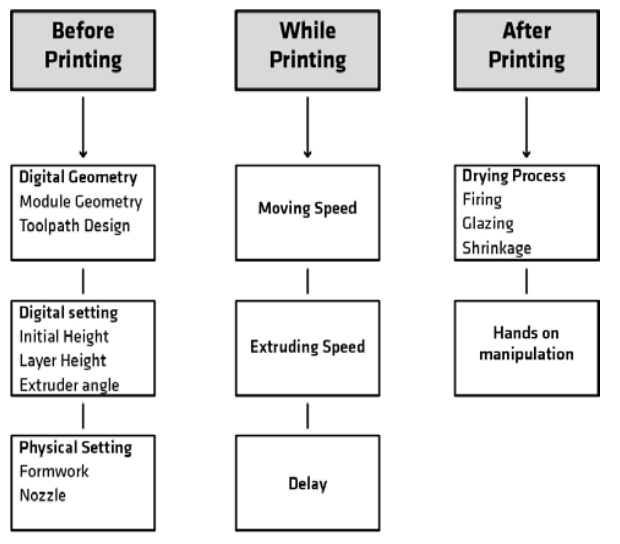

Figure 4: Printing parameters categorized based on their relationship with the production process

\section{TOP-DOWN DESIGN EXPLORATION}

A top-down design process is developed in parallel to the bottom-up explorations. This enabled to define the form and function of the project, and it also guided the bottomup explorations.

To begin with, three base modules are defined: simple extrusions of a triangle, rectangle and a hexagon. Different compositions of these three modules can tessellate a surface completely or partially. As it is shown in Figure 5, a planar surface can be tessellated only by repeating each base module or by combining two or three different modules. Modules can be connected through their edges or faces.

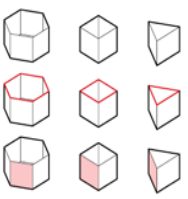

Figure 5: Base modules

\section{SYSTEMATIC EXPLORATIONS}

This section illustrates an example of sketching process within the desired workflow which involves the fabrication tools and techniques and emergent material behavior. The results of sketching are represented as set of systematic explorations to discover how to create different simple or complex objects in the process of making, using same digital design. In each set of exploration, one parameter has been changed while all other parameters have been kept constant. This enabled to systematically and comparatively explore the effects of robot's settings, clay types, digital inputs and real-time manipulations of digital setting on the final product.

\section{EXTRUSION SPEED}

Figure 6 illustrates the results of printing the same toolpath with different extrusion speeds. In the first two sample shown, objects show the noticeable difference in the wall thickness and clay bead section as a result of changing the speed. The object at the top row is printed with a lower extrusion speed rate than the object at the second row. This observation lead to creating the next two rows in Figure 6 . Third and fourth rows show different results of 3D printing the same toolpath as the first row by changing the extrusion speed from high to low in each layer from bottom to top of the object. The fifth row shows objects 3D printed with two specific extrusion speeds applied on every couple of layers.
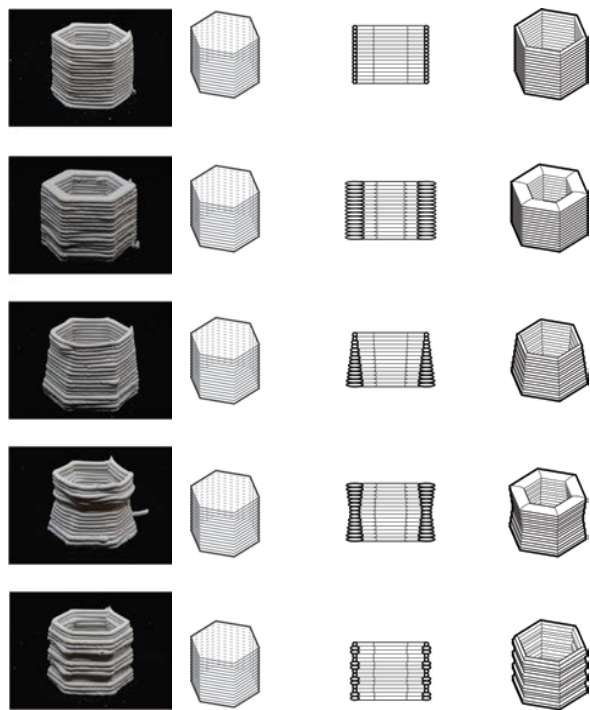

Figure 6: Physical models generated from the same toolpath with different extrusion speeds 


\section{EXTENSION LINE}

In previous experiments a curling effect was observed in the first couple of layers of the 3D printed objects. The reason for this is that at the start of the print the stepper motor needs some time to reach the defined speed of extrusion and the first printed line is a thin layer of clay, which leaves a gap between the actual layer height and printed layers. To eliminate this effect an extension line can be added to the toolpath of the first line in the first layer to give extra time for the stepper motor to reach the defined speed before starting to 3D print the actual object. Figure 7 illustrates two objects $3 \mathrm{D}$ printed following the same toolpath, one with the added extension line, and one without an extension line. The object on the left is 3D printed without an extension line. As can be seen, the first couple of layers are affected by the curling effect to fill the gap between the nozzle height and 3D printed clay. The object on the right, however, has cleaner layer definitions.
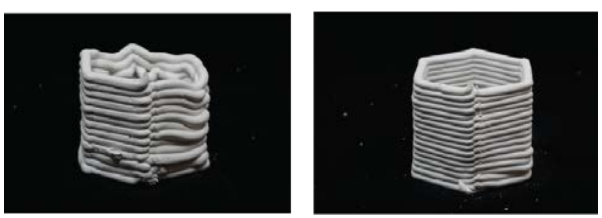

Figure 7: Left: object 3D printed without an extension line, Right: object 3D printed with an extension line.

\section{NOZZLE DIAMETER}

In Figure 8 the effects of the nozzle diameter are illustrated. First two rows show the results of 3D printing with a nozzle of $4 \mathrm{~mm}$ diameter and the second two rows show the same toolpath 3D printed with a nozzle of $3 \mathrm{~mm}$ diameter. As can be seen in Figure 8, smaller nozzle diameter makes the product unstable. Wall thickness becomes thinner, and layers cannot properly stick to one another, which results in collapsing of the higher printed layers.
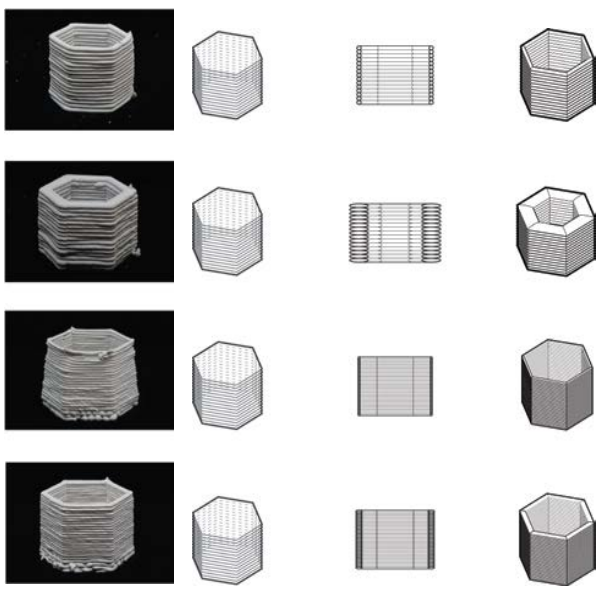

Figure 8: Same toolpaths 3D printed with different nozzle diameters

\section{NOZZLE HEIGHT}

Nozzle height is the distance between the $3 D$ printing surface and the nozzle. Changes in nozzle height can make the clay curl. When the distance between the nozzle and the printing surface is smaller than the nozzle diameter, clay bead gets squeezed and the layers can stick. However, when this distance is equal or higher than the nozzle diameter, the extruded clay bead has more space to change form before sitting on the previous layers. These effects are depicted in Figure 9 in which the nozzle height is gradually increased from the start to end of the toolpath in each layer. Increase in the differences in nozzle height also increases the curling effect and changes the geometry and texture of the 3D printed product.
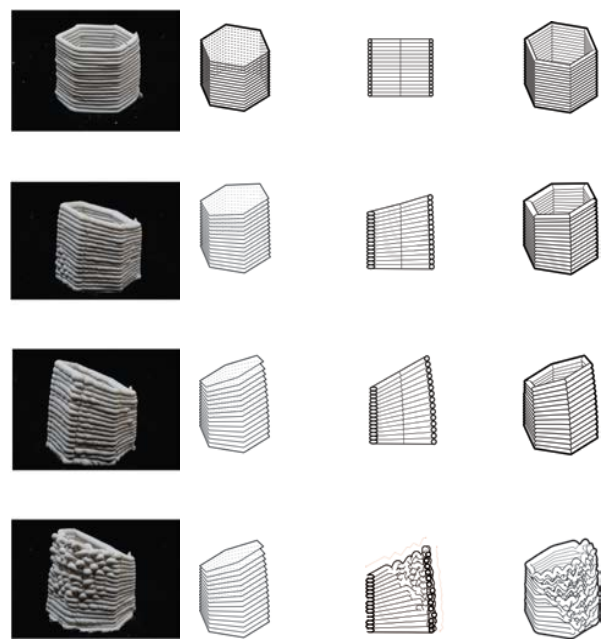

Figure 9: Gradual increase of nozzle height changes the surface texture

\section{TOOLPATH MODIFICATION}

Figure 10 shows the effects of manipulating the toolpath. From left to right, each layer is gradually rotated along the center of the object. Along with modifying the main geometry, this also enables to play with the surface texture.
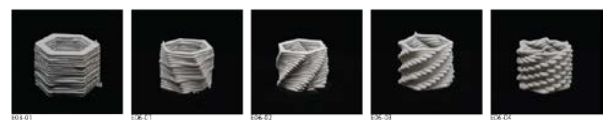

Figure 10: Gradual rotation of toolpath on each layer changes the surface texture

\section{CONCLUSION}

This project represents the first steps in exploring the potentials and limitations of digital fabrication tools and material properties as design tools. Defining a workflow in which designer, material and machines interact with each other to create new design ideas.

The example experiments of this research show that emergent effects of clay behavior as material in the process of robotic 3D printing can be a source of inspiration for designing and making complex geometries. Starting with simple abstract designs in the digital environment, it is possible to create a variety of physical objects by changing the tool and material parameters in the process of making. 

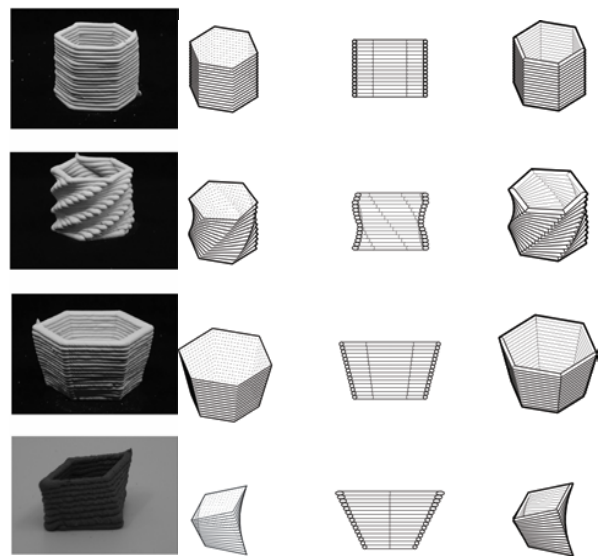

Figure 11: rotation, scale and deformation of toolpath on each layer

The workflow of this work shows the relationship between the tool, material, and designer (Figure 12). In the context of this research, tools are the robotic arm and the mechanical paste extruder with a software platform to connect them all together and to give the designer enough control over the process. Material exploration is limited to clay, to show case the sketching process in this workflow.

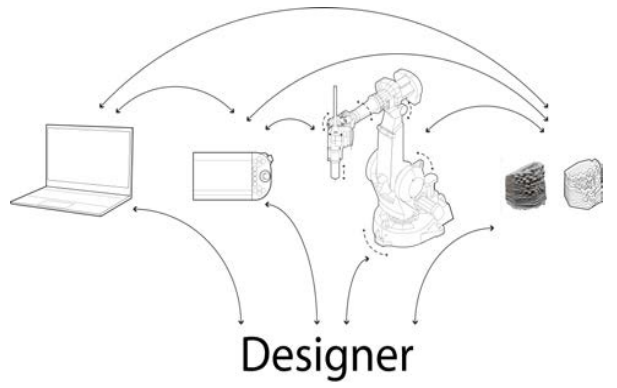

Figure 12: Robotic sketching workflow

Human agents and machine agents can achieve things together that neither one of them can achieve alone. The use of digital fabrication tools, and specifically robots in architecture and design opened up new paths that enabled to process materials faster with more accuracy. If the designers' thinking, decision-making, and interpretation of the process can also be integrated into the fabrication process, then the use of these machines can feed their creative processes as a means of sketching in design.

Similar to exploring design alternatives through drawings during a conventional sketching process, it is possible to explore alternatives with the matter itself, and even more so during the digital fabrication process. In this paper, one such example of sketching in the physical context using a robotic arm is shown. The robotic sketching process is exemplified following a systematic, and almost scientific approach. There are many uncertainties in a typical digital fabrication process that we usually do not take into consideration, and often consider as failures (Gursoy, 2018). In order to uncover these aspects and integrate them to our designs, we need to be able to repeat them. While a systematic approach enables us to document the emergent effects of material behavior, it can also give us a clearer sense of how different fabrication parameters affect the result. Documenting the results of the sketching process can allow us to recreate what we discovered while sketching. Moreover, this can enable the development of specific digital interfaces for generative design or simulations.

The question one may ask at this point is: What would happen when there are no unknowns in the robotic sketching process anymore? Why this workflow is defined as a feedback loop and not a linear process? To answer this question, it is necessary to highlight that the whole process is based on the designer's interpretation of the process and changes are made based on real-time decisions of the designer. Therefore, there will always be uncertainties, unknowns based on what the designer choses to see at any given time during the robotic sketching process. This subjectivity is what differentiates it from a sole scientific inquiry and renders it as a creative one.

\section{ACKNOWLEDGMENTS}

This paper is part of the MS thesis research of Paniz Farrokhsiar that is funded by the Pennsylvania State University, Department of Architecture, under the supervision of Benay Gursoy. The authors would to express their gratitude to the thesis committee members Jose Pinto Duarte, Felecia Davis and Tom Lauerman for their support and comments in the process of developing this research, and Ardavan Bidgoli, Jamie Heilman and Ardeshir Talaei for their knowledgeable advices and technical supports in the robotics area.

\section{REFERENCES}

Anton, A., \& Abdelmahgoub, A. (2018). Ceramic ComponentsComputational Design for Bespoke Robotic 3D Printing on Curved Support. 36th eCAADe Conference - Volume 2.

Bonwetsch, T. (2015). Robotically assembled brickwork: Manipulating assembly processes of discrete elements. Doctoral dissertation. ETH Zurich.

Cannaerts, C. (2009). Models of/Models for Architecture: Physica and Digital Modelling in Early Design Stages. eCAADe 27, (pp. 781-786). Istanbul.

Cera, B. (2018, 07 Oct). Mechanical Paste Extruder. Retrieved from MakerBot logo: https://www.thingiverse.com/thing:3142561

Cohen, Z. (2018). Hold Up: Machine Delay in Architectural Design. Robotic Fabrication in Architecture, Art and Design (pp. 126138). Cham: Springer.

Emerging Objects. (2015). Cool Clay. Retrieved from Emerging Objects: http://www.emergingobjects.com/project/cool-brick/

Emerging Objects. (2016). GCODE.Clay. Retrieved from Emerging Objects: http://www.emergingobjects.com/project/gcode-clay/

Emerging Objects. (2016). Seed Stitches. Retrieved from Emerging http://www.emergingobjects.com/project/seed-stitch/

Friedman, J., Kim, H., \& Mesa, O. (2014). Woven Clay. ACADIA

Gursoy, B. (2018). From Control to Uncertainty in 3D Printing with Clay. eCAADe.

Ingold, T. (2009). The textility of making. Cambridge journal of Economics 34, no. 1, 91-102. 
Jeremy, B., Norman, B., Rosenwasser, D., Liu, J. L., \& Sabin, J. (2018). ROBOSENSE 2.0 : Robotic Sensing and Architectural Ceramic Fabrication.

Lange, C., Holohan, D., \& Kehne, H. (2017). Ceramic INformation Pavilion. Rob|Arch (pp. 103-112). Springer.

Lange, C., Holohan, D., \& Kehne, H. (2018). Ceramic Constellation| Robotically Printed Brick Specials. Robotic Fabrication in Architecture, Art and Design. Cham (pp. 43444). Springer

Lawson, B., \& Menezes, A. (2006). How designers perceive sketches. Design Studies, $v 27,571-585$.

Pye, D. (1968). The nature and art of workmanship. London: Herbert Press.
Rosenwasser, D., Mantell, S., \& Sabin, J. (2017). Clay nonwovens: robotic fabrication and digital ceramics. ACADIA.

Seibold, Z., Hinz, K., del Castillo y López, J., Alonso, N. M., \& Mhatre, S. (2018). Ceramic Morphologies. Precision and control in paste-based additive manufacturing. ACADIA. , , , and Martin Bechthold.

Shi, J., Cho, Y., Taylor, M., \& Corre, D. (2019). Guiding InstabilityA craft-based approach for modular $3 \mathrm{D}$ clay printed masonry screen units. eCAADeS/GraDi.

Tokac, I., Bruyninckx, H., Cannaer, C., \& Moere, A. (2019). Material Sketching: Towards the Digital Fabrication of Emergent Material Effects. Extended Abstracts of the 2019 $\mathrm{CHI}$ Conference on Human Factors in Computing Systems, (pp. 1-6). 